\title{
SERVICIOS INNOVADORES EN LAS BIBLIOTECAS PÚBLICAS DE COLOMBIA. RESULTADOS DE UN ESTUDIO DELPHI
}

\author{
Innovative services in public libraries in Colombia. \\ Results of a Delphi study
}

Arley Soto y Sandra Suescún
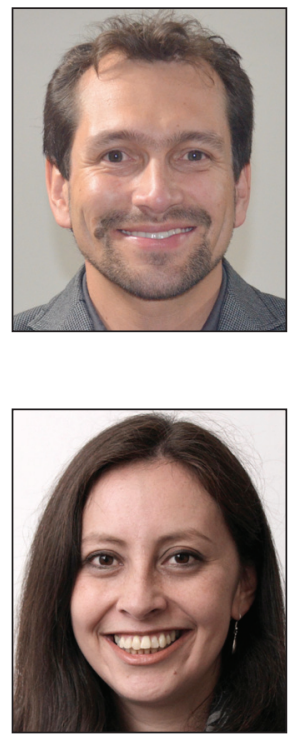

Arley Soto es master en bibliotecas digitales por las Universidades de Oslo \& Akershus, Tallin y Parma. Especialista en gerencia de proyectos, consultor en bibliotecas digitales, públicas y académicas. Co-fundador y gerente de proyectos de la empresa Biteca Ltda. Investiga en las áreas de bibliotecas públicas, preservación digital y publicación científica. En 2014 fue consultor de la Red Nacional de Bibliotecas Públicas de Colombia y formuló la metodología del modelo de madurez que se utiliza para la mejora de la calidad de los servicios. Es editor asociado del directorio de revistas de acceso abierto DOAJ. http://orcid.org/0000-0002-5878-2602

Av. Caracas, 34-86, of. 402, Bogotá, D.C., Colombia arleysoto@gmail.com

Sandra Suescún es profesional en ciencias de la información -bibliotecóloga- de la Pontificia Universidad Javeriana. Ha trabajado en coordinación de bibliotecas públicas, escolares y móviles, así como en las áreas de servicios bibliotecarios, promoción de lectura y escritura y capacitación. Es coordinadora nacional de servicios bibliotecarios de la Red Nacional de Bibliotecas Públicas (RNBP) en la Biblioteca Nacional de Colombia. Coordina la implementación del modelo de madurez de los servicios bibliotecarios para la RNBP y del Sistema de Información Nacional de usuarios y usos de las bibliotecas públicas Llave del Saber (2013 - 2015).

http://orcid.org/0000-0001-8125-0631

Calle 24, 5-60, 2으, Bogotá, D.C., Colombia ssuescun@mincultura.gov.co

\section{Resumen}

Se realizó un estudio Delphi con el objetivo de identificar tendencias de servicios innovadores en las bibliotecas públicas de Colombia mediante una consulta a expertos. Se realizaron tres rondas de consulta mediante cuestionarios estructurados online. Se encontraron cuatro grandes tendencias: fortalecimiento de las relaciones con la comunidad; convivencia entre libros, tecnología y usuarios; espacios para la creatividad y la comunicación y trabajo colaborativo y en red. Se concluye que la innovación de los servicios tiene que ver con la capacidad de la biblioteca de adaptarse a las condiciones de su entorno y la necesidad de una reflexión y acción permanente sobre los métodos, resultados e impacto de los servicios que ofrece la biblioteca pública.

\section{Palabras clave}

Bibliotecas públicas; Innovación; Servicios bibliotecarios; Estudios Delphi; Tendencias; Futuro; Prospectiva; Colombia.

\section{Abstract}

A Delphi study was performed to identify trends about innovative services in Colombian public libraries. An expert panel participated in three rounds with on-line questionnaires. Four trends were found: stronger community relationships; coexistence of books, technology and users; spaces for creativity, communication, collaborative work and networking. The study concluded that innovation at a public library is the capability to adapt services within its context and constantly engage in the necessary reflection and action about the methods, results and impact of its services.

\section{Keywords}

Public libraries; Innovation; Library services; Delphi studies; Trends; Future; Prospective; Colombia.

Soto, Arley; Suescún, Sandra (2015). "Servicios innovadores en las bibliotecas públicas de Colombia. Resultados de un estudio Delphi". El profesional de la información, v. 24, n. 6, pp. 778-786.

http://dx.doi.org/10.3145/epi.2015.nov.09 


\section{Introducción}

La innovación de los servicios en las bibliotecas públicas ha sido una preocupación creciente en los últimos años, principalmente debido a la generalización del uso de las tecnologías de la información y comunicación y su incidencia en la oferta de servicios de las bibliotecas. La facilidad con que las personas acceden ahora a la información que antes estaba solamente en documentos impresos ha exigido a las bibliotecas diseñar nuevos servicios y adaptarse a los cambios de la sociedad, especialmente cuando algunas de ellas sufren problemas de apoyo y sostenibilidad financiera (Gallo-León, 2015).

En las bibliotecas públicas la innovación está íntimamente relacionada con los procesos de aprendizaje y con la capacidad de ofrecer servicios de información y formación que contribuyan a resolver problemas identificados de la comunidad. Además, es preciso que responda a las exigencias de los cambios en su entorno recurriendo a procesos de innovación en sus técnicas de organización del conocimiento y en los servicios bibliotecarios (Lopera-Lopera, 2004).

\section{Creatividad e innovación en las bibliotecas públicas}

Aunque no toda la innovación tiene que ver con tecnología, en la época actual cuando la mayoría de información circula en medios digitales, muchos de los servicios innovadores están basados en tecnología. García-Gómez (2013) indica que la innovación en bibliotecas públicas se puede clasificar en tres grandes grupos:

- basada en las tecnologías de la información y de la participación:

- como soporte a su función sociocultural, caracterizada por su adaptabilidad al entorno, flexibilidad, experimentación y por su capacidad de romper patrones;

- como implicación en su comunidad, en la que se evidencia la capacidad de las bibliotecas públicas para buscar usuarios potenciales de todo tipo y brindar servicios de información específicos para comunidades particulares, tanto en las instalaciones de la biblioteca como fuera de ella.

La innovación en las bibliotecas públicas

es relativa. Lo que es innovador en un contexto específico puede no serlo en otro

La innovación en las bibliotecas públicas es relativa. Lo que es innovador en un contexto específico puede no serlo en otro, ya que las necesidades y condiciones de las comunidades pueden ser diferentes. Esta relatividad de la innovación exige un proceso constante de análisis del contexto, de pensamiento creativo y un ambiente flexible, pero planeado y de gestión eficaz que genere cambios que permitan prestar más y mejores servicios para los usuarios reales y potenciales.

En el ámbito organizacional, la innovación contempla múltiples significados:

- creación (invención);

- difusión y aprendizaje;

- suceso;
- trayectoria (corriente de innovaciones);

- cambio (creciente o radical);

- proceso o estrategia;

- innovación a nivel de contexto.

Hay un elemento común en todos ellos y es la capacidad de un proceso para agregar valor a cualquier actividad o resultado específicos (Ahmed et al., 2012).

Siguiendo el concepto anterior, en el sector de las bibliotecas públicas la innovación tiene que ver con la capacidad de éstas de agregar valor a sus servicios y espacios para contribuir a resolver problemas identificados en la comunidad.

La definición más sencilla de innovación en bibliotecas públicas quizás sea la formulada por Lozano-Díaz (2008), quien afirma que "la verdadera innovación implica que lo que se ha implantado (con base tecnológica o no) sean productos, servicios o prácticas útiles para el ciudadano, que consigan la mejora de la calidad de los servicios, y de la imagen de la biblioteca" y lo ha resumido en una simple frase: "algo nuevo, algo útil, algo de calidad", a lo que podemos agregarle que puede ser en términos del servicio, del proceso y/o de la estrategia.

Es indispensable que los nuevos procesos, estrategias o servicios fortalezcan la inclusión y contribuyan a disminuir la brecha digital e informacional

Todo proceso de innovación y de gestión tecnológica en una biblioteca, ha de tener en cuenta el derecho de los ciudadanos al acceso a la información y al asesoramiento en su uso (Lopera-Lopera, 2004). Por lo tanto es indispensable que los nuevos procesos, estrategias o servicios fortalezcan la inclusión y contribuyan a disminuir la brecha digital e informacional.

Existe un triángulo de conexiones entre incertidumbre, riesgo y creatividad; de hecho una tolerancia saludable al riesgo y a la incertidumbre pueden estimular el pensamiento creativo (Anderson, 2011). No obstante, la creatividad y la innovación suponen un esfuerzo por parte de quienes están a cargo de las bibliotecas, ya que la creatividad necesita tiempo: para pensar, para analizar, para abstraer, para compartir con otros, para discutir y debatir.

Para Walton (2008) la creatividad es una estrategia para que las bibliotecas desempeñen nuevos servicios en un mundo con cambios turbulentos. Aunque la creatividad e innovación no siempre son compatibles con la necesidad de incrementar la eficiencia, mejorar la calidad y alcanzar la productividad en las bibliotecas, estas se pueden desarrollar a través de varias estrategias de gestión informadas y cohesivas.

Por su parte, Sheng y Sun (2007) definen la cultura de la innovación del conocimiento como una clase de valor, comportamiento y sistema institucional que consigue ventajas competitivas y desarrollo sostenible de las bibliotecas a través de la creación de conocimiento. Los autores enfatizan que la cultura de la innovación se fomenta a través de estrategias de gestión de conocimiento. 


\section{Estudio Delphi}

El método Delphi tiene como propósito

"obtener el mayor consenso confiable de la opinión de un grupo de expertos (...) mediante una serie de cuestionarios intensivos intercalados con retroalimentaciones de opinión controladas" (Dalkey; Helmer, 1963).

En esta investigación se siguieron las siguientes reglas para un estudio Delphi sugeridas por Pickard (2013):

- en el panel sólo participan expertos;

- todos los datos se recogen en forma escrita;

- se intenta sistemáticamente producir un consenso;

- se realizan al menos dos rondas;

- se mantiene el anonimato de los expertos mientras se realiza.

Algunas de las ventajas de esta técnica de investigación es que ofrece un adecuado canal para penetrar en áreas de conocimiento complejas, dinámicas, ambiguas y con falta de información, siendo el coste de obtención de la información bajo y su tiempo en principio reducido (Ortega, 2008).

En la bibliotecología se ha utilizado el estudio Delphi en varias investigaciones, pero quizá una de las más relevantes en los últimos años ha sido la presentada por Ludwig y Starr (2005). A partir de un cuestionario con preguntas abiertas sobre los posibles cambios futuros en los roles e instalaciones de las bibliotecas de salud y en cómo los cambios en la tecnología podrían afectar el espacio de las bibliotecas en el futuro, se diseñaron un total de 200 afirmaciones que permitieron realizar las posteriores rondas de consenso entre los expertos.

También es conocido el estudio realizado por Pickard, Gannon-Leary y Coventry (2010). A partir de un formulario con preguntas abiertas se recibieron comentarios que sirvieron para construir un modelo sobre criterios de confiabilidad para recursos de información en la Web, que fue debatido en una mesa redonda entre expertos. Recientemente en España el grupo estratégico para el estudio de prospectiva sobre la biblioteca en el nuevo entorno informacional y social, realizó una consulta a expertos utilizando elementos Delphi para llevar a cabo el estudio Prospectiva 2020: las diez áreas que más van a cambiar en nuestras bibliotecas en los próximos años (Consejo de Cooperación Bibliotecaria, 2013).

\section{Metodología}

El estudio Delphi se realizó durante el primer semestre de 2014. El propósito de la investigación fue identificar las principales tendencias de servicios en las bibliotecas públicas de Colombia, para lo cual se plantearon los siguientes objetivos específicos:

- recopilar opiniones de diversos expertos sobre la innovación de servicios en las bibliotecas;

- categorizar consensos y no consensos entre los expertos y analizar los resultados de la categorización;

- formular posibles recomendaciones para la aplicación en la Red Nacional de Bibliotecas Públicas de Colombia, a corto, medio y largo plazo.
En el estudio participaron 17 expertos colombianos. La selección se realizó teniendo en cuenta la trayectoria, experiencia e idoneidad en el área de bibliotecas públicas y/o innovación. Se invitó a:

- bibliotecólogos y otros profesionales con experiencia en la prestación de servicios en bibliotecas públicas;

- bibliotecólogos a cargo de empresas comerciales;

- profesores universitarios de facultades de ciencias de la información;

- profesionales de otras áreas con trayectoria en las áreas de innovación, servicios y bibliotecas públicas.

Las etapas de la investigación fueron las siguientes:

\section{Paso 1. Cuestionario 1. Preguntas abiertas}

Cuestionario de cinco preguntas abiertas enviado a un primer grupo de expertos. Se invitó a responder de manera libre y espontánea, con el fin de que se pudiera recoger la mayor cantidad de información, para luego elaborar los formularios con preguntas controladas.

\section{Paso 2. Cuestionario 2. Ronda 1}

A partir de las opiniones recogidas en el paso anterior, se diseñó un cuestionario online con 55 enunciados. Por cada enunciado los expertos marcaron su grado de acuerdo o desacuerdo en cuatro escalas tipo Likert en relación con la probabilidad de cambio, deseabilidad de cambio, certeza de la respuesta y el año en el que ocurrirá el cambio.

\section{Paso 3. Cuestionario 3. Ronda 2}

Se sacaron del cuestionario aquellos enunciados donde se identificó claramente un consenso entre los expertos. Las afirmaciones restantes se revisaron y se elaboró un nuevo cuestionario indicando los argumentos de los expertos a favor y en contra. Cada experto contestó el nuevo cuestionario y considerando los argumentos expuestos se le pidió nuevamente que marcara su grado de acuerdo o desacuerdo en las mismas escalas tipo Likert del cuestionario 2. Al final se realizó un focus group (grupo de discusión) con los expertos para discutir los enunciados donde no hubo consenso.

\section{Resultados}

Los expertos consideran unas condiciones previas que debería tener una biblioteca pública para innovar en sus servicios:

- Capacidad de adaptación al entorno: las bibliotecas deberán conocer el entorno y las necesidades de su comunidad, para lo cual realizarán estudios de usuarios periódicamente y diseñarán programas y servicios acordes con estas necesidades.

- Recursos humanos competentes y suficientes: son la condición fundamental para que las bibliotecas públicas se transformen en espacios dinámicos. Por lo tanto la presencia permanente de personal bibliotecario creativo, suficiente y competente permitirá a la biblioteca posicionarse, moverse y transformarse en la medida que cambia el entorno.

- Apropiación de tecnología: los cambios en la tecnología permitirán tener más servicios, contenidos y llegar a más 
Tabla 1. Consensos tendencia 1

\begin{tabular}{|c|c|c|c|}
\hline Acuerdo(\%) & Enunciado & Probabilidad & Tiempo \\
\hline 99 & $\begin{array}{l}\text { Las BP contribuirán significativamente a la construcción de la historia local de las comunida- } \\
\text { des y a la memoria colectiva de los pueblos mediante de la publicación de estos contenidos } \\
\text { en internet }\end{array}$ & $\begin{array}{l}\text { Definitivamente } \\
\text { realizable }\end{array}$ & $2015-2020$ \\
\hline 99 & $\begin{array}{l}\text { Las BP fortalecerán su relación con universidades y grupos especializados para llevar a cabo } \\
\text { procesos de investigación en la biblioteca (ej. semilleros de investigación, proyectos de inves- } \\
\text { tigación...) }\end{array}$ & Muy probable & $2015-2025$ \\
\hline 98 & $\begin{array}{l}\text { Las BP serán los principales espacios para recibir orientación sobre oportunidades de desarro- } \\
\text { llo para la comunidad (becas, empleo, pasantías, educación gratuita, beneficios del gobierno, } \\
\text { subsidios, trámites, vivienda, salud, bienestar, etc.) }\end{array}$ & Muy probable & 2015-2025 \\
\hline 98 & $\begin{array}{l}\text { Las BP crearán nuevas formas de interacción entre los usuarios y el encuentro de las personas } \\
\text { a través de la tecnología. }\end{array}$ & $\begin{array}{l}\text { Definitivamente } \\
\text { realizable }\end{array}$ & $2015-2025$ \\
\hline 96 & $\begin{array}{l}\text { Las BP diseñarán sus programas y servicios con la participación de la comunidad, a través de } \\
\text { mecanismos personalizados o virtuales como laboratorios o talleres, entre otros }\end{array}$ & Muy probable & $2020-2025$ \\
\hline 94 & $\begin{array}{l}\text { Las BP deberán generar e incentivar directamente la creación de comunidades de aprendizaje } \\
\text { interdisciplinares y nuevos espacios de diálogo e intercambio entre los usuarios }\end{array}$ & Muy probable & $2020-2025$ \\
\hline 93 & $\begin{array}{l}\text { Las BP ofrecerán servicios de alerta a través de SMS u otra tecnología dirigida a dispositivos } \\
\text { móviles para comunicar las novedades, recomendaciones y programación de la biblioteca y } \\
\text { de la localidad }\end{array}$ & $\begin{array}{l}\text { Definitivamente } \\
\text { realizable }\end{array}$ & $2015-2025$ \\
\hline 86 & $\begin{array}{l}\text { Las BP llevarán a cabo investigaciones sobre el acceso a la información, su impacto e implica- } \\
\text { ciones sociales }\end{array}$ & Muy probable & $2015-2025$ \\
\hline 85 & Las BP ofrecerán formación sobre privacidad y protección de datos & $\begin{array}{l}\text { Definitivamente } \\
\text { realizable }\end{array}$ & $2015-2020$ \\
\hline
\end{tabular}

personas. Las bibliotecas tendrán la posibilidad de brindar mayor oferta de servicios y contenidos y apoyarse en la tecnología para que sus usuarios accedan sin limitaciones de tiempo y espacio a información de calidad, lo que permitirá mayores posibilidades de visibilidad y llegar a más personas en distintos lugares.

- Las bibliotecas públicas ofrecerán servicios descentralizados, incluyentes e interculturales: el servicio de extensión bibliotecaria continuará siendo una prioridad, ofreciendo espacios, servicios y programas que permitan que todas las personas tengan la oportunidad de usar la biblioteca (servicios a poblaciones rurales, adultos mayores, minorías étnicas, personas con discapacidad, desplazados, extranjeros, inmigrantes, etc.).

Estas condiciones previas deberán aumentar la capacidad de la biblioteca para llegar a más personas.

Los resultados del estudio se han agrupado en cuatro grandes tendencias:

- biblioteca como espacio de aprendizaje comunitario;

- convivencia y complementariedad entre libros, tecnología y comunidad;

- concepción de espacios para la creatividad y la comunicación;

- gestión colaborativa y trabajo en red.

\section{Tendencia 1. Biblioteca como espacio de aprendizaje comunitario}

Los expertos consideran que las bibliotecas públicas (BP) son espacios privilegiados de aprendizaje a lo largo de toda la vida. Las bibliotecas crearán nuevas formas de interacción entre los usuarios y posibilitarán que las personas participen en espacios de creación y aprendizaje colectivo. Los expertos están de acuerdo en que algunos servicios presenciales se convertirán en servicios remotos, pero no todos, y advierten sobre la inconveniencia de ver la tecnología como un fin en sí misma. Consideran que la biblioteca es un lugar de encuentro y un espacio de aprendizaje e integración; por lo tanto las actividades y servicios como talleres, actividades culturales, programas de promoción de lectura y formación seguirán siendo una prioridad y se continuarán prestando presencialmente. De la misma manera reconocen que teniendo en cuenta la diversidad de usuarios y las brechas sociales, educacionales, y/o tecnológicas de la comunidad, será necesario el rol formador del bibliotecario (tabla 1).

\section{Tendencia 2. Convivencia entre libros, tecnología y comunidad}

Los expertos consideran que las tecnologías, los libros y las personas van a convivir más cercanamente en las bibliotecas mediante la creación de servicios y espacios que promuevan nuevas formas de relación e interacción entre ellos.

Reconocen que para este propósito es necesario tener más y mejores libros, mejor tecnología y una mayor velocidad de conexión a internet. Los expertos están de acuerdo en que el servicio de préstamo externo tradicional de material físico no será reemplazado por el acceso remoto a libros, música y películas online, porque las condiciones de desigualdad social y económica del contexto colombiano no lo permiten (tabla 2). 
Tabla 2. Consensos tendencia 2

\begin{tabular}{|c|c|c|c|}
\hline Acuerdo (\%) & Enunciado & Probabilidad & Tiempo \\
\hline 99 & $\begin{array}{l}\text { Todas las BP ofrecerán internet gratuito y de alta velocidad donde los usuarios puedan reali- } \\
\text { zar la tareas normales, subir, descargar y editar contenidos }\end{array}$ & Muy probable & $2015-2020$ \\
\hline 99 & $\begin{array}{l}\text { Las BP contarán con espacios cómodos y adecuados a los distintos usos y poblaciones, así } \\
\text { como salas flexibles para distintos usos: lectura individual, salas para trabajos colectivos y } \\
\text { salas para la creación }\end{array}$ & Probable & $2020-2025$ \\
\hline 98 & Las BP ofrecerán espacios especializados para jóvenes & Muy probable & $2015-2030$ \\
\hline 94 & $\begin{array}{l}\text { Las BP transformarán el enfoque de la biblioteca de un espacio controlado y vigilado a un } \\
\text { espacio en el que las personas se sientan libres y satisfechas }\end{array}$ & Probable & $2015-2030$ \\
\hline 93 & $\begin{array}{l}\text { Los servicios tradicionales personalizados que ofrecen las BP se mantendrán al tiempo que } \\
\text { se implementan servicios especializados, novedosos y online }\end{array}$ & $\begin{array}{l}\text { Definitivamente } \\
\text { realizable }\end{array}$ & $2015-2020$ \\
\hline 91 & $\begin{array}{l}\text { El servicio de préstamo externo de material físico seguirá funcionando, pero se combinará } \\
\text { con el préstamo de libros, música y películas online }\end{array}$ & $\begin{array}{l}\text { Definitivamente } \\
\text { realizable }\end{array}$ & $2015-2025$ \\
\hline 91 & Las BP ofrecerán salas de lectura digital o virtual online & $\begin{array}{l}\text { Definitivamente } \\
\text { realizable }\end{array}$ & 2020-2025 \\
\hline 87 & $\begin{array}{l}\text { Las BP ofrecerán tutoriales sobre el uso de la biblioteca en general y sobre cada uno de sus } \\
\text { servicios }\end{array}$ & $\begin{array}{l}\text { Definitivamente } \\
\text { realizable }\end{array}$ & $2015-2025$ \\
\hline 87 & $\begin{array}{l}\text { Las tecnologías, los libros y las personas van a convivir más cercanamente, sin necesidad de } \\
\text { establecer espacios de uso especial para las TIC }\end{array}$ & Muy probable & $2015-2025$ \\
\hline 86 & $\begin{array}{l}\text { Las BP desarrollarán mecanismos virtuales que complementen los servicios y programas } \\
\text { presenciales (ej. foros, clubes de lectura, charlas, cursos online, videoconferencias, programas } \\
\text { culturales, entre otros por internet) }\end{array}$ & $\begin{array}{l}\text { Definitivamente } \\
\text { realizable }\end{array}$ & $2015-2020$ \\
\hline 85 & Las BP ofrecerán variadas posibilidades de auto-servicio & $\begin{array}{l}\text { Definitivamente } \\
\text { realizable }\end{array}$ & $2015-2025$ \\
\hline 84 & $\begin{array}{l}\text { Las BP ofrecerán préstamo de materiales a domicilio (entrega y devolución de materiales } \\
\text { físicos en el lugar de residencia o trabajo del usuario) }\end{array}$ & Poco probable & $2015-2025$ \\
\hline
\end{tabular}

Tabla 3. Consensos tendencia 3

\begin{tabular}{|c|c|c|c|}
\hline Acuerdo (\%) & Enunciado & Probabilidad & Tiempo \\
\hline 99 & $\begin{array}{l}\text { Las BP ofrecerán un buen espacio de lectura o simplemente para charlar, con menos escritorios } \\
\text { y más sillas cómodas y espacios frescos y luminosos }\end{array}$ & Muy probable & $2015-2030$ \\
\hline 98 & $\begin{array}{l}\text { Las BP ofrecerán tecnologías complementarias para la producción y edición de contenidos por } \\
\text { parte de los usuarios }\end{array}$ & Muy probable & $2020-2025$ \\
\hline 98 & $\begin{array}{l}\text { Las BP ofrecerán formación para el uso y apropiación de programas tecnológicos (software, } \\
\text { edición, diseño, etc.), según intereses de los usuarios }\end{array}$ & Muy probable & 2020-2025 \\
\hline 98 & $\begin{array}{l}\text { Las BP se reestructurarán administrativamente, de manera que los bibliotecarios puedan dedi- } \\
\text { carse más a la creación de nuevos servicios que a acciones meramente instrumentales como el } \\
\text { procesamiento técnico y el préstamo de materiales }\end{array}$ & Probable & 2020-2025 \\
\hline 96 & $\begin{array}{l}\text { Las BP crearán tiempos y espacios que promuevan la creatividad y la generación de ideas y } \\
\text { proyectos por parte de los equipos bibliotecarios }\end{array}$ & Probable & $2020-2025$ \\
\hline 93 & $\begin{array}{l}\text { Las BP posibilitarán que las personas razonen de forma colectiva, creen y participen colectiva- } \\
\text { mente, y que por este camino encuentren soluciones a problemas individuales o comunes }\end{array}$ & Muy probable & $2020-2025$ \\
\hline 92 & $\begin{array}{l}\text { Las BP ofrecerán espacios que impulsen la apropiación tecnológica y la creación de aplicacio- } \\
\text { nes por parte de los usuarios con el uso de software comercial y libre }\end{array}$ & Muy probable & $2015-2025$ \\
\hline 92 & $\begin{array}{l}\text { Las BP crearán espacios físicos y condiciones para el estímulo y desarrollo del pensamiento } \\
\text { creativo, la innovación y el emprendimiento a partir del uso y la experimentación con las TIC, y } \\
\text { que permitan la construcción colectiva de ideas y de saberes (ej. salas de innovación, laborato- } \\
\text { rios, aulas didácticas, salas abiertas) }\end{array}$ & Muy probable & $2020-2025$ \\
\hline 92 & $\begin{array}{l}\text { Las BP formarán equipos interdisciplinares con diversos perfiles profesionales que se ajusten a } \\
\text { la nuevas realidades }\end{array}$ & Muy probable & 2020-2025 \\
\hline 87 & $\begin{array}{l}\text { Las BP crearán contenidos libres online (que puedan reusarse, modificarse y usarse incluso con } \\
\text { fines comerciales, según criterio del creador) }\end{array}$ & Muy probable & 2020-2025 \\
\hline 87 & $\begin{array}{l}\text { Las BP priorizarán procesos de formación en escritura y creación de contenidos, especialmente } \\
\text { en los medios digitales }\end{array}$ & Muy probable & 2020-2025 \\
\hline
\end{tabular}


Tabla 4. Consensos tendencia 4

\begin{tabular}{|c|c|c|c|}
\hline Acuerdo (\%) & Enunciado & Probabilidad & Tiempo \\
\hline 99 & $\begin{array}{l}\text { Las BP generarán procesos de articulación con otras bibliotecas y entidades que compartan } \\
\text { sus principales intereses y propósitos, ya sea en espacios físicos o virtuales, para compartir } \\
\text { experiencias, conocimientos y generar proyectos conjuntos }\end{array}$ & Muy probable & 2015-2025 \\
\hline 99 & $\begin{array}{l}\text { Las BP documentarán y sistematizarán experiencias de proyectos y programas que permitan } \\
\text { enriquecer las prácticas de otras bibliotecas }\end{array}$ & Muy probable & 2015-2020 \\
\hline 98 & $\begin{array}{l}\text { Las BP dejarán de trabajar de manera individual y aislada. Los cambios en la tecnología cam- } \\
\text { biarán las lógicas de trabajo de las bibliotecas públicas, generando nuevas lógicas colaborati- } \\
\text { vas, multidisciplinarias, interinstitucionales y distribuidas }\end{array}$ & Muy probable & 2015-2020 \\
\hline 98 & $\begin{array}{l}\text { Las BP participarán activamente en espacios de socialización de experiencias innovadoras } \\
\text { para exponer los proyectos que realizan con sus respectivas comunidades }\end{array}$ & $\begin{array}{l}\text { Definitivamente } \\
\text { realizable }\end{array}$ & 2015-2020 \\
\hline 96 & $\begin{array}{l}\text { La tecnología impactará permanentemente los servicios de las bibliotecas, haciéndolos más } \\
\text { eficientes porque permitirá mayor oportunidad en la respuesta, ahorrar tiempos, optimizar } \\
\text { recursos, gestionar con agilidad las inquietudes de los usuarios, inferir necesidades de infor- } \\
\text { mación }\end{array}$ & Muy probable & 2020-2025 \\
\hline 96 & $\begin{array}{l}\text { Las BP realizarán encuentros regionales y nacionales que estimulen la creación de nuevos } \\
\text { programas y servicios entre bibliotecas }\end{array}$ & Muy probable & $2015-2020$ \\
\hline 95 & $\begin{array}{l}\text { Las BP promoverán un ambiente de trabajo que permita procesos de innovación y creatividad } \\
\text { (trabajo en equipo, colaboración, creación de lazos entre personas, entre bibliotecas y entre } \\
\text { instituciones) }\end{array}$ & Muy probable & $2020-2025$ \\
\hline 93 & $\begin{array}{l}\text { Las bibliotecas participarán activamente en convocatorias de eventos que reconozcan, visibili- } \\
\text { cen y premien las ideas y proyectos innovadores en materia de bibliotecas públicas }\end{array}$ & Muy probable & 2015-2020 \\
\hline 93 & $\begin{array}{l}\text { Las redes de BP realizarán consorcios o convenios para la adquisición de bases de datos y } \\
\text { plataformas de préstamo de libros digitales }\end{array}$ & Muy probable & 2020-2025 \\
\hline 91 & $\begin{array}{l}\text { Las redes BP ofrecerán infraestructuras de gestión de conocimiento (intranets, sistemas de } \\
\text { gestión de conocimiento y redes de conocimiento bibliotecario) }\end{array}$ & Probable & 2020-2025 \\
\hline 86 & $\begin{array}{l}\text { Las BP ofrecerán acceso online a bases de datos en todas las áreas del conocimiento y colec- } \\
\text { ciones digitales de calidad mediante consorcios, alianzas y negociaciones colectivas con los } \\
\text { proveedores }\end{array}$ & Probable & 2020-2025 \\
\hline 84 & $\begin{array}{l}\text { Las BP ofrecerán el servicio de referencia mediante mecanismos que no requieran la presencia } \\
\text { (chat, correo electrónico, teléfono, sms...) a través de consorcios u otras estrategias colabora- } \\
\text { tivas }\end{array}$ & Muy probable & 2015-2025 \\
\hline
\end{tabular}

\section{Tendencia 3. Espacios para la creatividad y la comu- nicación}

Los expertos están de acuerdo en que las bibliotecas públicas contarán con espacios multipropósito que además de favorecer la lectura individual, posibiliten la creación y la comunicación entre las personas. Estos nuevos espacios propondrán otras posibilidades de diálogo entre la comunidad, así como condiciones para el desarrollo y estímulo del pensamiento creativo, la innovación y el emprendimiento (tabla 3).

\section{Tendencia 4. Gestión colaborativa y trabajo en red}

Los expertos están de acuerdo con que las bibliotecas públicas dejarán de trabajar de manera individual y aislada. Al contrario, requieren generar estrategias de gestión colaborativa y multidisciplinar, así como trabajo en red con instituciones de diversa índole. Lo anterior supone una participación activa de la biblioteca pública en el contexto bibliotecario y aprovechar su potencial de liderazgo comunitario (tabla 4).

\section{Desacuerdos y no consensos}

En la tabla 5 se presentan los enunciados en donde hubo consenso en contra del enunciado y aquellos en donde no hubo consenso entre los expertos; es decir, que a pesar de que se hizo una segunda ronda con estos enunciados, indicando los argumentos de los expertos a favor y en contra, no se logró consenso.

Otros servicios basados principalmente en la tecnología generan posiciones a favor y en contra por parte de los expertos. Por ejemplo, no todos están de acuerdo con que las bibliotecas creen programas de formación virtual no formal a corto o mediano plazo, porque existen otras instituciones que cumplen esa función, y además requieren de cierta infraestructura para ofrecerlos con calidad. No obstante, quienes están a favor consideran que es una oportunidad para la biblioteca a largo plazo. Tampoco ven probable que la biblioteca pública se oriente al desarrollo de hardware y software, con argumentos como que resulta muy costoso y que otros espacios cumplen esta función, pero sobre todo porque la biblioteca pública en América Latina tiene otras prioridades, y que distraer recursos para asumir otros roles sería un riesgo para su sostenibilidad y estabilidad.

Los expertos ven aún más difícil que las relaciones entre la comunidad y la biblioteca sean solamente a través de las TIC, así como poco probable que los servicios presenciales se transformen en servicios remotos. La posición general se puede resumir con la afirmación textual de uno de los 
expertos: "Mientras las bibliotecas sean espacios físicos las relaciones sociales directas y presenciales se mantendrán y se fortalecerán en tanto cada vez se reconocen más por la contribución que hacen en el territorio para el fortalecimiento de las metas sociales y comunitarias".

\section{Los expertos ven difícil que las relaciones entre la comunidad y la biblioteca sean solamente a través de las TIC y que los servicios presenciales se transformen en servicios remotos}

\section{Conclusiones}

La necesidad de que las bibliotecas públicas ofrezcan internet de alta velocidad para que los usuarios puedan subir, descargar, consultar y editar contenidos, es la tendencia que ha tenido mayor consenso entre los expertos.

Sin embargo, al preguntarles sobre la posibilidad de que las bibliotecas eliminen los bloqueos para consultar ciertos sitios web, éstos consideran que aunque sería ideal la no existencia de censura para la consulta de información, la presencia de menores de edad en la biblioteca le concede una responsabilidad sobre ellos. Por tanto en la práctica será necesario establecer mecanismos tecnológicos para el bloqueo de ciertos sitios web inadecuados para los menores de edad.

Asimismo se enfatiza la importancia de la formación a los usuarios para que tengan una posición crítica frente a la tec- nología, así como la necesidad de una política consensuada para el acceso a internet en las bibliotecas públicas, de manera que si existe alguna restricción para consultar sitios web, responda a dicha política y no dependa del punto de vista personal del bibliotecario.

Sobre el préstamo de materiales físicos para su consulta en casa, los expertos coinciden en que seguirá funcionando a corto, medio y largo plazo. Todos son muy escépticos acerca de que el préstamo de materiales online vaya a reemplazar completamente el préstamo tradicional. No obstante, reconocen la importancia de ofrecer acceso remoto a bases de datos, libros, música y películas online y agregan que el suministro de estos contenidos debe realizarse preferiblemente a través de consorcios a nivel nacional o regional, con el fin de reducir costos así como tener mayor cobertura.

\section{Los expertos son muy escépticos acerca} de que el préstamo de materiales online vaya a reemplazar completamente el préstamo tradicional

En relación con el préstamo a domicilio (entrega y devolución de materiales físicos en el lugar de residencia o trabajo del usuario), consideran que aunque sería deseable y probable a medio plazo, resulta costoso y difícil de administrar, por lo tanto sugieren implementarlo principalmente para personas con discapacidad o imposibilidad de desplazarse a la biblioteca o implementar estrategias de préstamo online si la comunidad dispone de acceso a internet.

Tabla 5. Desacuerdos y no consensos

\begin{tabular}{|c|c|c|c|}
\hline Acuerdo (\%) & Enunciado & Probabilidad & Tiempo \\
\hline 78 & Las BP crearán programas de formación virtual no formal & Probable & 2015-2025 \\
\hline 78 & $\begin{array}{l}\text { Las BP llevarán a cabo modelos de gestión que incluyan sistemas de incentivos a la creación y } \\
\text { un tipo de atmósfera tolerable a los fallos }\end{array}$ & Improbable & 2020-2025 \\
\hline 71 & $\begin{array}{l}\text { Las BP quitarán cualquier bloqueo de acceso a la información (restricciones para consultar } \\
\text { ciertas páginas web, filtros de contenido...) }\end{array}$ & Poco probable & 2020-2025 \\
\hline 68 & $\begin{array}{l}\text { Las BP perfilarán los servicios de acuerdo con la ocupación e intereses de los usuarios más que } \\
\text { por grupos de edad }\end{array}$ & Muy probable & 2015-2020 \\
\hline 68 & Las BP ofrecerán cursos virtuales y presenciales de cómo armar una biblioteca digital personal & Muy probable & 2020-2025 \\
\hline 54 & $\begin{array}{l}\text { Las BP se orientarán al desarrollo de hardware libre y software de código abierto (que puedan } \\
\text { reusarse, modificarse y usarse incluso con fines comerciales) }\end{array}$ & Poco probable & 2025-2030 \\
\hline 33 & $\begin{array}{l}\text { Las BP establecerán relaciones con la comunidad sólo a través de la mediación con las TIC, los } \\
\text { social media, la web } 2.0 \text {, las publicaciones digitales y los contenidos electrónicos }\end{array}$ & Improbable & - \\
\hline 32 & Las BP irán transformando todos los servicios presenciales en servicios de acceso remoto & Improbable & - \\
\hline 12 & $\begin{array}{l}\text { La tecnología anula las barreras de espacio y de tiempo para acceder a la información, hacien- } \\
\text { do innecesaria la presencia de intermediarios como las BP }\end{array}$ & Improbable & - \\
\hline 11 & $\begin{array}{l}\text { El servicio de referencia dejará de funcionar y será reemplazado por un buen programa de } \\
\text { alfin }\end{array}$ & Improbable & \\
\hline 9 & $\begin{array}{l}\text { El servicio de préstamo externo de material físico dejará de funcionar y será reemplazado por } \\
\text { acceso remoto a libros, música y películas online }\end{array}$ & Improbable & - \\
\hline
\end{tabular}


El servicio de referencia en las bibliotecas públicas se irá transformando en la medida que la comunidad vaya cambiando. Los expertos coinciden en que las comunidades requerirán personal bibliotecario con mejores competencias, así como un rol formador dentro de sus comunidades. Aunque a medio o largo plazo este servicio puede además prestarse con mecanismos que no requieran la mediación presencial (chat, correo electrónico, teléfono, sms...) será fundamental contar con referencistas con alto nivel de formación para atender las posibles consultas de los usuarios. Una vez más la tecnología en este caso es simplemente un medio y el éxito de su implementación dependerá de la capacidad humana para ofrecer un servicio de calidad.

La tecnología es un medio y el éxito de la implementación de un servicio de referencia remoto dependerá de la capacidad humana para ofrecer un servicio de calidad

La aplicación de nuevas tecnologías en las bibliotecas públicas permitirá mejorar su gestión e impactará permanentemente los servicios de las bibliotecas, haciéndolos más eficientes porque permitirá mayor rapidez de los procesos, así como ahorrar tiempo y optimizar recursos. Los expertos consideran que además de mejorar los servicios existentes, la implementación de nuevas tecnologías permitirá crear nuevos servicios así como la oferta de variadas posibilidades de auto-servicio. Las posibilidades de aplicación de nuevas tecnologías con más consenso son:

- en la construcción de la historia local de las comunidades a través de diferentes medios;

- creación de nuevas formas de interacción y encuentro entre usuarios;

- formación para la apropiación de programas tecnológicos (software, edición, diseño...), según intereses de los usuarios;

- servicios para comunicar las novedades, recomendaciones y programación de la biblioteca y de la localidad;

- disponibilidad de espacios que impulsen la apropiación tecnológica y la creación de aplicaciones por parte de los usuarios con el uso de software comercial y libre.

La innovación de los servicios tiene que ver con la capacidad de la biblioteca de adaptarse a las condiciones de su entorno y la necesidad de una reflexión y acción permanente sobre los métodos, resultados e impacto de los servicios que ofrece. Una de las condiciones previas es contar con recursos humanos competentes y suficientes, así como una rápida apropiación de los cambios tecnológicos y sociales en su comunidad, para ofrecer servicios descentralizados, incluyentes e interculturales.

Las principales tendencias en Colombia son:

- concepción de la biblioteca pública como un espacio de aprendizaje comunitario en donde se prioriza el rol formador del bibliotecario;

- convivencia entre libros, tecnología y comunidad, para lo cual es necesario una buena infraestructura arquitectónica y conectividad;
- espacios para la creatividad y la comunicación que promuevan la capacidad creativa de los usuarios;

- gestión colaborativa y trabajo en red, condición fundamental para fortalecer su liderazgo en la comunidad y optimizar recursos que le permitan aumentar la calidad y cobertura de sus servicios.

Finalmente, los expertos coinciden en que la biblioteca pública como espacio e institución social seguirá vigente y con una gran responsabilidad frente a la disminución de brechas existentes en la sociedad, con una oferta de servicios tradicionales de calidad fortalecidos con el uso y aprovechamiento de la tecnología, así como nuevos servicios que consideren los constantes cambios que se dan en cada contexto.

\section{Nota}

Este trabajo fue patrocinado parcialmente por el proyecto de uso y apropiación de TIC, Convenio Ministerio de Cultura de Colombia - Fundación Bill \& Melinda Gates.

\section{Agradecimientos}

Los autores expresan un sincero agradecimiento a todos los expertos que participaron en el estudio Delphi sobre servicios innovadores:

Adriana Betancur, Adriana Ordóñez, Ana Roda, Andrea López, Catalina Holguín, Carolina Delgado, David Ramírez, Diana Yucumá, Didier Álvarez, Gloria-María Rodríguez, Henry García, Janeth Ardila, José-Ignacio Caro, Juan-Carlos Sierra, Leonardo Machett, Mary Giraldo, Orlanda Jaramillo.

Expertos que contribuyeron al diseño metodológico:

Logan Ludwig y Susan Starr.

\section{Bibliografía}

Ahmed, Pervaiz K.; Shepherd, Charles; Ramos-Garza, Leticia; Ramos-Garza, Claudia (2012). Administración de la innovación. México: Pearson Educación. ISBN: 9786073208550

Anderson, Theresa D. (2011). "Beyond eureka moments: supporting the invisible work of creativity and innovation". Information research, v. 16, n. 1.

http://InformationR.net/ir/16-1/paper471.html

Consejo de Cooperación Bibliotecaria (2013). Prospectiva 2020: las diez áreas que más van a cambiar en nuestras bibliotecas en los próximos años. Madrid: Ministerio de Educación, Cultura y Deporte. Gobierno de España.

$h t t p: / / g o o . g l / r f b w A O$

Dalkey, Norman; Helmer, Olaf (1963). "An experimental application of the Delphi method to the use of experts". Management science, v. 9, n. 3. pp. 458-467.

http://dx.doi.org/10.1287/mnsc.9.3.458

Gallo-León, José-Pablo (2015). "La biblioteca es servicio (y en ello está nuestro futuro)". El profesional de la información, v. 24, n. 2, pp. 87-93.

http://www.elprofesionaldelainformacion.com/ contenidos/2015/mar/01.pdf

http://dx.doi.org/10.3145/epi.2015.mar.01

García-Gómez, Francisco-Javier (2013). “Innovación en la 
biblioteca pública española: dónde estamos y hacia dónde vamos". Revista general de información y documentación, v. 23, n 1. pp. 133-150.

http://dx.doi.org/10.5209/rev_RGID.2013.v23.n1.41449

Lopera-Lopera, Luis-Hernando (2004). "Las interacciones entre información e innovación desde la perspectiva de una ética bibliotecológica". En: $1^{\text {er }}$ Foro social de información, documentación y bibliotecas, Buenos Aires (Argentina), 2628 Agosto.

http://eprints.rclis.org/6579

Lozano-Díaz, Roser (2008). "Innovación en bibliotecas públicas: algo nuevo, algo útil, algo de calidad". El profesional de la información, v. 17, n. 2. pp. 129-134.

http://recyt.fecyt.es/index.php/EPI/article/view/epi.2008. mar.01

http://dx.doi.org/10.3145/epi.2008.mar.01

Ludwig, Logan; Starr, Susan (2005). "Library as place: Results of a Delphi study". Journal of the Medical Library Association, v. 93, n. 3, pp. 315-326.
http://www.ncbi.nlm.nih.gov/pmc/articles/PMC1175798/

Ortega, Félix (2008). "El método Delphi: prospectiva en ciencias sociales a través del análisis de un caso práctico". Revista Escuela de Administración de Negocios, pp. 31-54.

Pickard, Alison-Jane (2013). Research methods in information ( $2^{\text {nd }}$ ed.). Londres: Facet London. ISBN: 9781856048132

Pickard, Alison-Jane; Gannon-Leary, Pat; Coventry, Lynne (2010). Users' trust in information resources in the Web environment: a status report. JISC Final report. Londres: JISC. http://nrl.northumbria.ac.uk/729

Sheng, Xiaoping; Sun, Lin (2007). "Developing knowledge innovation culture of libraries". Library management, v. 28, n. 1-2, pp. 36-52.

http://dx.doi.org/10.1108/01435120710723536

Walton, Graham (2008). "Theory, research, and practice in library management 4: Creativity". Library management, v. 29, n. 1-2, pp. 125-131.

http://dx.doi.org/10.1108/01435120810844702

\section{Revistas de comunicación, documentación y biblioteconomía indexadas en el Social Sciences Citation Index, de Thomson Reuters, que aceptan artículos en español Impact factor 2014}

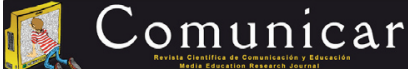

0,838 Comunicar (España) http://www.revistacomunicar.com

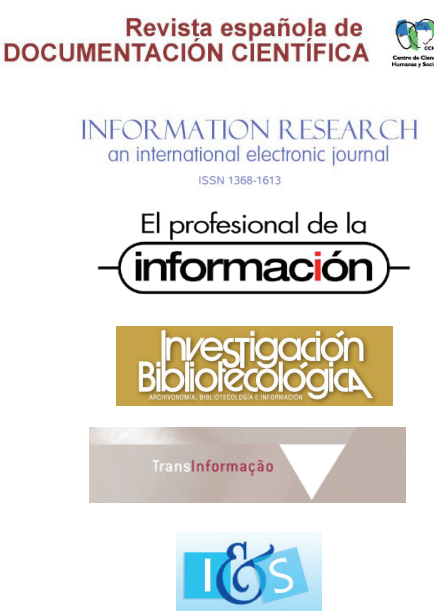

0,636 Revista española de documentación científica (España) http://redc.revistas.csic.es/index.php/redc

0,370 Information research (Reino Unido) http://informationr.net/ir

0,356 El profesional de la información (España) http://www.elprofesionaldelainformacion.com

0,114 Investigación bibliotecológica (México) http://www.revistas.unam.mx/index.phplibi

0,095 Transinformação (Brasil) http://www.scielo.br/scielo.php?script=sci_serial\&pid=0103-3786

0,082 Informação \& sociedade: estudos (Brasil) http://www.ies.ufpb.brlojs2/index.phplies

\section{Situación de otras revistas}

Perspectivas em ciência da informação (Brasil) http://portaldeperiodicos.eci.ufmg.brlindex.php/pci Sin IF desde 2013. En 2015 no aparece indexada en la WoS

\section{Comunicación y sociedad (España)}

http://www.unav.es/fcom/comunicacionysociedad/es

Tuvo IF los años 2008-2012. En octubre de 2014 inició una nueva etapa publicando en inglés

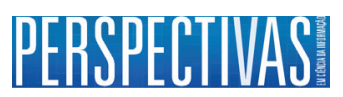

COMMUNICATION \& SOCIETY

Nota: Existe otra revista Comunicación y sociedad (México) http://www.comunicacionysociedad.cucsh.udg.mx Indexada en la WoS desde 2008. No se le asigna IF porque es una de las casi 700 revistas de SCielo integradas en la WoS en enero de 2014

Estudios sobre el mensaje periodístico (España)

http://revistas.ucm.es/index.php/ESMP

Indexada 2008-2011, IF en 2010-2012 